Saccucchi, E. y Avila Castro, M.P. Análisis de discurso de la judicialización de cuatro conflictos ambientales en Córdoba, Argentina. Derecho y Ciencias Sociales. Mayo - Octubre 2020. № 23 .Pgs 1-22 ISNN 1852-2971. Instituto de Cultura Jurídica y Maestría en Sociología Jurídica. FCJ y S. UNLP

\title{
Análisis de discurso de la judicialización de cuatro conflictos ambientales en Córdoba, Argentina
}

Discourse Analysis of the judicialization of four environmental conflicts in Córdoba, Argentina

\author{
Erika Saccucci• \\ María Paula Ávila Castro*•
}

\section{Resumen}

La profundización de los conflictos frente al avance del agronegocio ha supuesto nuevos campos y modos de lucha para los movimientos socioambientales, así como transformaciones en el derecho ambiental. En el marco de nuestras sociedades neoliberales, esto puede comprenderse como un proceso de judicialización de las luchas, en las que se produce una revalorización de lo judicial como medio de arbitraje de los conflictos, frente al protagonismo de la dinámica de las empresas que elevan la conflictividad social en el ejercicio de sus actividades. En este artículo abordamos cuatro conflictos socioambientales de la provincia de Córdoba (Argentina) con el foco puesto en lo que concebimos como prácticas judiciales. Por medio del análisis de discurso de diversos recursos jurídicos interpuestos por los miembros de estos colectivos, estudiamos dos ejes: uno de contenido ambiental, vinculado al principio precautorio; otro formal, relacionado con la puesta en juego de tres argumentos procedimentales: los estudios de impacto ambiental, las audiencias públicas y las disposiciones de uso del suelo. Nuestros primeros análisis sugieren que los colectivos en lucha invocan un uso estratégico del derecho, más allá de los delitos ambientales, que está más próximo al efecto legitimante de la evidencia científica y a aspectos formales, administrativos o de regulaciones de otro tipo de materia no ambiental, cuyo incumplimiento puede presentarse de manera clara y contundente frente a las formas de enunciación y apropiación ideológicas del derecho ambiental por parte de las autoridades jurídicas.

Palabras clave: agronegocio - conflictos - judicialización - neoliberalismo- discurso.

\begin{abstract}
The deepening of conflicts related to agribusiness has involved new fields and ways of struggle for social and environmental groups, as well as transformations in the environmental law. In our neoliberal societies, this can be understood as a process of judicialization of the struggles, which means there is a revaluation of the judiciary
\end{abstract}

\footnotetext{
- Erika Saccucci es Doctora en Estudios Sociales de América Latina. Correo: erika_sac34@hotmail.com; Orcid: http://orcid.org/0000-0002-2469-0988.Docentes e investigadoras en la Universidad Católica de Córdoba, Argentina e integrantes del Colectivo de Investigación "El Llano en Llamas” www.llanocordoba.com.ar”

- María Paula Ávila Castro es Magíster en Sociología. Correo: mapaulaac@gmail.com; Orcid: https://orcid.org/0000-0003-1915-9284. Docentes e investigadoras en la Universidad Católica de Córdoba, Argentina e integrantes del Colectivo de Investigación "El Llano en Llamas" www.llanocordoba.com.ar"
}

Recibido: 1/9/2019 Publicable con correcciones: 22/6/2020 
Saccucchi, E. y Avila Castro, M.P. Análisis de discurso de la judicialización de cuatro conflictos ambientales en Córdoba, Argentina. Derecho y Ciencias Sociales. Mayo - Octubre 2020. № 23 .Pgs 1-22 ISNN 1852-2971. Instituto de Cultura Jurídica y Maestría en Sociología Jurídica. FCJ y S. UNLP

power as arbitrating conflicts over the protagonism of companies that elevates social conflict in the exercise of their activities. In this article we approach four social and environmental conflicts in the province of Córdoba (Argentina) with focus on what we conceive as judicial practices. Through discourse analysis of various legal devices presented by the members of these struggling groups, we study two aspects: an environmental matter related to the precautionary principle; and a formal matter related to the use of three procedural arguments: environmental impact studies, public audiences and land use dispositions. Our first conclusions suggest that struggle groups invoke a strategic use of law beyond environmental crime, which is closer to the legitimatory effect of scientific evidence and to formal, administrative or other non-environmental regulations aspects, whose failure to comply is more clearly and forcefully presented to the forms of ideological enunciation and appropriation of the legal authorities.

Key words: agribusiness - conflicts - judicialization - neoliberalism- discourse. 
Saccucchi, E. y Avila Castro, M.P. Análisis de discurso de la judicialización de cuatro conflictos ambientales en Córdoba, Argentina. Derecho y Ciencias Sociales. Mayo - Octubre 2020. № 23 .Pgs 1-22 ISNN 1852-2971. Instituto de Cultura Jurídica y Maestría en Sociología Jurídica. FCJ y S. UNLP

\title{
Análisis de discurso de la judicialización de cuatro conflictos ambientales en Córdoba, Argentina
}

\author{
Erika Saccucci y María Paula Ávila Castro
}

\section{Introducción}

En las últimas décadas se registra un avance del agronegocio en la provincia de Córdoba. Se trata de una de las principales productoras de soja y otros cereales tales como el maíz y el trigo del país. De acuerdo con la información disponible, en la provincia el porcentaje de superficie plantada con soja aumentó de 22,9\% a 42,2\% en el período que va de 1988 a 2002, mientras que el cultivo de cereales pasó de 20,2 a 25,4\% en el mismo período y territorio (do Carmo y Álvarez, 2009).

Esto ha generado una particular conflictividad ambiental a partir del surgimiento de una pluralidad de organizaciones, vecinas y vecinos autoconvocados y asambleas en defensa del ambiente y la salud (Alonso y otros, 2015). A su vez, la profundización de los conflictos socioambientales y la inclusión de la problemática del ambiente como una temática de relevancia política ha supuesto modificaciones en las normativas que lo regulan y la incorporación de la lucha jurídica como una estrategia en el marco de los conflictos (Villegas Guzmán, 2018).

En esta investigación nos enfocamos en cuatro conflictos socioambientales de la provincia de Córdoba, relacionados con el agronegocio: la histórica lucha de las Madres de Barrio Ituzaingó Anexo por las fumigaciones en las inmediaciones del barrio; la defensa de la ordenanza que prohíbe el uso de agrotóxicos en áreas próximas a la ciudad de Alta Gracia; la movilización contraria a la radicación de una planta de acopio de semillas de la multinacional Monsanto en la localidad de Malvinas Argentinas; y la lucha contra la permanencia de la fábrica de Porta Hnos. que produce bioetanol y alcohol en el corazón del barrio San Antonio de la ciudad de Córdoba.

En estas experiencias de lucha resalta la interposición de acciones legales como amparos, demandas o intervenciones tanto en los ámbitos administrativos como penales del sistema judicial; ya sea por iniciativa de los colectivos en lucha o bien como respuesta a la judicialización de los casos por parte del poder político o económico. Estas estrategias jurídicas se vinculan, a veces en consonancia y otras en contradicción, con otro tipo de estrategias no 
Saccucchi, E. y Avila Castro, M.P. Análisis de discurso de la judicialización de cuatro conflictos ambientales en Córdoba, Argentina. Derecho y Ciencias Sociales. Mayo - Octubre 2020. № 23 .Pgs 1-22 ISNN 1852-2971. Instituto de Cultura Jurídica y Maestría en Sociología Jurídica. FCJ y S. UNLP

jurídicas tales como movilizaciones, bloqueos, intervenciones artísticas, ferias, talleres o festivales.

Una revisión somera de investigaciones en las que se da tratamiento a estas problemáticas, nos ofrece diversos abordajes sobre la relación del derecho ambiental y las luchas sociales. Por una parte, encontramos aquellos estudios que proponen que los derechos ambientales son definidos, apropiados y exigidos por actores sociales y estatales a través de un abanico variado de formas de acción (Gutiérrez, 2015). La participación social ayuda a posicionar las cuestiones ambientales en la agenda pública (Downs, 1972; Harrison, 1996) a la vez que funciona como un mecanismo democrático para el control social de las decisiones de las élites económicas y políticas (Peruzzotti y Smulovitz, 2002). En esta mirada también se actualizan y resignifican los derechos ambientales, tanto por su incidencia en la definición pública (Offe, 1992) como en la definición jurídico-legal de esos derechos (Azuela, 2006). Este enfoque resulta interesante, aunque supone una perspectiva de tipo pluralista en la que se equipara a los diversos actores sociales como grupos de interés que traccionan el derecho ambiental en diversos sentidos, de acuerdo con los grados de organización de cada uno y la efectividad de sus estrategias de acción. En esta "arena política" quedan desdibujadas las relaciones de poder asimétricas que se establecen entre unos y otros actores, así como las posibilidades de acceso a las estrategias disponibles.

Por otra parte, encontramos aquellas investigaciones (Saccucci, 2018; Villegas Guzmán, 2018; Alonso y otros, 2015; de la Vega, 2014) que conciben las luchas ambientales como expresiones de la conflictividad actual en oposición a un modelo de despojo y saqueo de los bienes comunes. Los conflictos en torno a la tierra y el ambiente en los últimos años encuentran fundamento en la creciente incorporación de territorios marginales dentro de las dinámicas de producción y circulación capitalista. Así, los diversos actores sociales no se encuentran en condiciones de igualdad, sino que las propias luchas dan cuenta de la desigualdad que ha sido efectuada por el accionar estatal. Al mismo tiempo, el Estado no es percibido como monolítico, sino que en él actúan lógicas y dispositivos que se solapan y contradicen entre sí. En este marco, el derecho no es pura fuerza, sino que se requiere complejizar el análisis a fin de reconocer y analizar las estrategias y prácticas que se organizan desde los sujetos sociales en conflicto para confrontar el orden y uso jurídico hegemónico.

Esta perspectiva, a su vez, sostiene que la contienda judicial funciona como una arena de legitimación simbólica para los actores en la que se debaten sentidos en torno a la justicia, el ambiente y las formas de habitar. El discurso jurídico es un "discurso altamente codificado, 
Saccucchi, E. y Avila Castro, M.P. Análisis de discurso de la judicialización de cuatro conflictos ambientales en Córdoba, Argentina. Derecho y Ciencias Sociales. Mayo - Octubre 2020. № 23 .Pgs 1-22 ISNN 1852-2971. Instituto de Cultura Jurídica y Maestría en Sociología Jurídica. FCJ y S. UNLP

expresado a través de símbolos tendientes al desplazamiento permanente de los conflictos hacia los lugares menos visibles" (Entelman, 1982, p.17). Lo discursivo no está formado sólo por palabras, sino -como afirma Cárcova- "es también comportamientos, símbolos, conocimientos, es lo que los jueces interpretan, los abogados implementan, los litigantes declaran, los teóricos producen, los legisladores sancionan y los doctrinarios critican" (citado por Landry, 2012, p.82).

En este artículo nos inscribimos dentro del segundo abordaje por considerar que los conflictos ambientales expresan tensiones en los modos de producción, contra el saqueo de los bienes comunes y que es preciso estudiarlos a través del análisis de los discursos que allí se configuran. Por ello, recuperando a De Sousa Santos (2000), coincidimos en que estas investigaciones deben aportar a desarrollar una conceptualización sociológica del derecho autónoma de la que ha sido elaborada por los juristas y por la ciencia jurídica para superar uno de los más persistentes obstáculos epistemológicos a la constitución de un objeto teórico propio de la sociología del derecho.

En esta investigación intentamos ahondar en ello y presentar una propuesta preliminar que vincula, en una misma perspectiva teórica, los conflictos ambientales y los procesos de judicialización de las luchas dentro de la dinámica neoliberal contemporánea, en relación con la importancia estratégica del saber científico para la legitimación de la lucha contra los efectos contaminantes del agronegocio.

En definitiva, en base al estudio del conjunto de instrumentos interpuestos por los colectivos en lucha de los casos seleccionados, llevamos adelante un ejercicio de indagación que pretende caracterizar el proceso de creciente judicialización de la lucha ambiental contra el agronegocio. La reflexión sobre estos aspectos nos propone desarrollar un análisis de discurso, entendido como el estudio crítico del nivel estratégico-político en el que se inscribe la lucha ambiental dentro del marco de las prácticas judiciales.

En el apartado 2. exponemos brevemente el encuadre teórico y metodológico de la presente investigación, orientado por un conjunto de reflexiones foucaultianas sobre el concepto de práctica judicial y el papel del derecho en la dinámica neoliberal. Quedarán explicitadas, a su vez, cuestiones generales sobre los conflictos bajo estudio. El análisis propuesto se desarrolla en el apartado 3., el cual explora el sentido estratégico de las prácticas judiciales del derecho ambiental relacionado con los casos seleccionados, alrededor de dos grandes ejes: uno de contenido ambiental, vinculado al principio precautorio; otro formal, relacionado con la puesta en juego de tres argumentos procedimentales: los estudios de impacto ambiental, las audiencias 
Saccucchi, E. y Avila Castro, M.P. Análisis de discurso de la judicialización de cuatro conflictos ambientales en Córdoba, Argentina. Derecho y Ciencias Sociales. Mayo - Octubre 2020. № 23 .Pgs 1-22 ISNN 1852-2971. Instituto de Cultura Jurídica y Maestría en Sociología Jurídica. FCJ y S. UNLP

públicas y las disposiciones de uso del suelo. En el apartado 4 ensayamos algunas reflexiones posibles.

\section{Perspectiva teórica y aspectos metodológicos}

\subsection{Las prácticas judiciales}

En las experiencias de lucha que abordamos han cobrado protagonismo la elaboración y presentación de amparos, cautelares, demandas penales, solicitudes administrativas, amicus curiae, entre otras, por medio de representantes legales como abogados/as privados/as o clínicas jurídicas de interés social. Este conjunto de prácticas se inscribe en lo que podemos denominar prácticas judiciales, esto es, procedimientos estipulados por el derecho ambiental argentino y provincial que regulan las consecuencias de ciertas actividades económicas con efectos en la naturaleza y la salud de las personas. Contemplan las responsabilidades civiles y penales que pueden derivarse, por ejemplo, de la aplicación de agrotóxicos, la producción de bioetanol o el acopio de semillas modificadas genéticamente en lo que respecta al agronegocio.

Estas prácticas judiciales no sólo pueden ser entendidas como modos formales de proceder entre las partes, sino como discursos que definen tipos de subjetividad y formas de saber específicas que, en palabras de Foucault, implican en rigor "relaciones entre el hombre y la verdad que merecen ser estudiadas" (Foucault, 2007a, p.16).

Un aspecto central de la práctica judicial desde su constitución es la importancia de la oposición de formas racionales de prueba y demostración: “cómo producir la verdad, en qué condiciones, qué formas han de observarse y qué reglas han de aplicarse" (Foucault, 2007a, p.66). Ello involucra una técnica específica, que es la indagación como modo de determinación de la verdad en el campo jurídico, una técnica que se ha difundido a otros dominios de saber y que se constituyó con el tiempo como forma general de saber en Occidente. Para Foucault, la indagación que es propia de la práctica judicial se coloca precisamente en la conjunción entre un tipo de poder y ciertos contenidos de conocimiento (Foucault, 2007a).

Los mecanismos y discursos que involucra el derecho a un ambiente sano en el marco nacional y provincial y en diferentes niveles, no escapan a esta configuración de las prácticas judiciales, en las que siempre es posible identificar el ejercicio de un poder específico, relacionado con procesos económico-políticos, y la definición y reproducción de un saber con efectos de sentido específicos respecto de la veracidad de los objetos que dirime el sistema jurídico. De ahí que cuando ponemos el foco en los conflictos bajo estudio emerjan, no sólo modalidades de ejercicio del poder vinculados al desarrollo del agronegocio que tienen que ver con la 
Saccucchi, E. y Avila Castro, M.P. Análisis de discurso de la judicialización de cuatro conflictos ambientales en Córdoba, Argentina. Derecho y Ciencias Sociales. Mayo - Octubre 2020. № 23 .Pgs 1-22 ISNN 1852-2971. Instituto de Cultura Jurídica y Maestría en Sociología Jurídica. FCJ y S. UNLP

factibilidad y profundización del desarrollo de ciertas actividades económicas y políticas, sino, y de la mano, disputas sobre la legitimidad de estas actividades que se argumentan en un plano científico respecto de la existencia de daño o responsabilidad sobre la salud de las personas y del ambiente en general.

Los conflictos relacionados con el agronegocio son siempre conflictos de saber, en los que hay una pretensión de demostración de verdad, por medio de las mencionadas técnicas de indagación de la práctica judicial. En palabras de Foucault: "La indagación es precisamente una forma política, de gestión, de ejercicio del poder que, por medio de la institución judicial, pasó a ser, en la cultura occidental, una manera de autentificar la verdad" (2007a, p.92).

Lo referido es todavía más patente en nuestras sociedades neoliberales, en las que es evidente una revalorización de lo judicial como medio de arbitraje de los conflictos, entre ellos los ambientales. Podríamos hablar en la actualidad de un creciente intervencionismo jurídico (Foucault, 2007b). Ello es posible en tanto el arte de gobierno neoliberal conlleva dos procesos: por un lado, un mayor protagonismo de la dinámica de las empresas, las cuales deben regirse por las relaciones de mercado sin intervención sobre las reglas de juego económicas por parte del poder político, que a su vez, elevan la conflictividad social en el ejercicio de sus actividades; por otro lado, y en concomitancia, un corrimiento de las instancias de acción administrativa del Estado y un incremento de la intervención judicial frente a esa conflictividad.

Este escenario nos permite comprender, en términos generales, la creciente judicialización de los conflictos, incluidos los relacionados con el agronegocio, y en particular, la significativa introducción de estrategias judiciales en la lucha de los colectivos socioambientales organizados que aquí conceptualizamos como prácticas judiciales.

En definitiva, el análisis de discurso que se puede llevar adelante sobre estas prácticas judiciales, refiere a la dimensión polémica y estratégica de los documentos bajo estudio. Se trata de un análisis del discurso como juego estratégico político de las prácticas judiciales (Foucault, 2007a).

En este sentido, comprendemos el discurso como un efecto de sentido entre locutores (Orlandi, 1994), a partir del cual es posible observar la relación entre lenguaje, historia e ideología. Para ello, nuestra indagación versa sobre un corpus de documentos legales implicados en los casos (detallados en la bibliografía), a partir de una metodología cualitativa de análisis del discurso. A continuación, realizamos una descripción de los conflictos estudiados.

\subsection{Conflictos contra el agronegocio en el Gran Córdoba}


Saccucchi, E. y Avila Castro, M.P. Análisis de discurso de la judicialización de cuatro conflictos ambientales en Córdoba, Argentina. Derecho y Ciencias Sociales. Mayo - Octubre 2020. № 23 .Pgs 1-22 ISNN 1852-2971. Instituto de Cultura Jurídica y Maestría en Sociología Jurídica. FCJ y S. UNLP

\subsubsection{Caso Madres de Barrio Ituzaingó Anexo}

A fines de 2001, un grupo de madres del Barrio Ituzaingó Anexo ${ }^{1}$ comenzó a preocuparse por la presencia de diversos casos de cáncer en la comunidad. Alertadas por la recurrencia de los casos en el barrio efectuaron relevamientos de personas enfermas casa por casa. Los resultados mostraron la presencia de 60 casos de cáncer sobre un total de 5000 habitantes.

Las vecinas organizadas, basadas en los datos del relevamiento, presentaron una denuncia en la Secretaría de Derechos Humanos y Medio Ambiente y en el Ministerio de Salud de la Nación, solicitando estudios de sedimentos de tanques, de suelo, de transformadores de luz de PBC, de aire y de campos magnéticos. Frente a la ausencia de respuestas por parte del Estado en marzo de 2002 salieron a la calle por primera vez para reclamar atención sanitaria ante la cantidad de personas enfermas en el barrio.

Medardo Ávila Vázquez, subsecretario de Salud de la Municipalidad de Córdoba en aquel entonces, realizó una denuncia por "envenenamiento" al ver cómo se fumigaba sobre las casas del barrio. El fiscal de Instrucción del Distrito III, Carlos Matheu, ordenó estudios en los patios de las viviendas y confirmó la presencia de endosulfán y glifosato. En base a ello determinó la figura penal de "contaminación dolosa del medio ambiente de manera peligrosa para la salud". En 2004 las Madres presentaron un amparo ambiental que llegó a juicio en la Cámara I del Crimen de Córdoba. Este juicio finalizó el 22 de agosto de 2012, cuando la Cámara emitió un fallo inédito para América Latina. Determinó que fumigar con agrotóxicos es delito. El caso del barrio Ituzaingó Anexo se convirtió en el primero en llegar a juicio penal y en el que un productor agropecuario y un aerofumigador fueron encontrados culpables por el delito de contaminación ambiental, sentenciándolos a una pena de tres años de prisión condicional.

En el año 2012 las Madres presentaron una nueva denuncia conocida como el juicio de la "Causa Madre". Se trata de una acusación que involucra a los productores por "contaminación" y supone la vinculación entre las fumigaciones, la contaminación y la presencia de enfermedades.

\subsubsection{Caso VUDAS/ Porta Hnos.}

La empresa Porta Hnos. es originariamente productora de alcohol, sin embargo, en el año 2012 incluyó dentro de sus actividades la producción de bioetanol. Es importante señalar que esta empresa es la única planta productora de bioetanol localizada dentro del ejido urbano, en el

\footnotetext{
${ }^{1}$ Ituzaingó Anexo es un barrio ubicado al sur de la ciudad de Córdoba, en la periferia, colindante con la zona industrial metal-mecánica y la frontera agrícola.
} 
Saccucchi, E. y Avila Castro, M.P. Análisis de discurso de la judicialización de cuatro conflictos ambientales en Córdoba, Argentina. Derecho y Ciencias Sociales. Mayo - Octubre 2020. № 23 .Pgs 1-22 ISNN 1852-2971. Instituto de Cultura Jurídica y Maestría en Sociología Jurídica. FCJ y S. UNLP

corazón de un barrio residencial. En 2013 los/as vecinos/as realizaron una denuncia penal contra la empresa por contaminación, la cual quedó a cargo del fiscal José Mana que ordenó una pericia científica. Esto resulta de relevancia en tanto era la primera vez que en el fuero penal se realizó una pericia para medir la contaminación en el aire.

En 2016 las vecinas presentaron un amparo ambiental ante el juez Bustos Fierro, el cual fue rechazado en septiembre de ese año. El amparo fue contra la empresa Porta y contra los Ministerios de Ambiente y Energía de la Nación. Ante la apelación de las vecinas, la Cámara Federal ordenó reabrir el caso y le derivó el expediente a Vaca Narvaja quien ordenó la primera audiencia de conciliación el día 7 de agosto de 2017, a la cual solo concurrieron las vecinas. Actualmente, el juicio se encuentra en una instancia indagatoria. Resulta necesario destacar que se trató del segundo caso de amparo ambiental en todo el país, luego del amparo de Madres de Barrio Ituzaingó.

\subsubsection{Caso Ordenanza sobre fumigaciones con agrotóxicos de la localidad de Alta Gracia}

En octubre de 2012 la Municipalidad de Alta Gracia sancionó la ordenanza municipal № 9.375 que establece una zona de resguardo ambiental de 1500 metros, la cual prohíbe la utilización en toda forma, de cualquier tipo de producto químico o biológico de uso agrícola. Esta ordenanza limitó las fumigaciones como consecuencia de la lucha de vecinas y vecinos de barrios periféricos de la ciudad (en especial de barrio Parque San Juan, ubicado en el extremo este de la localidad), que habían comenzado a denunciar problemas de salud y daño ambiental por la aplicación de agrotóxicos cerca de sus viviendas.

A poco de la sanción de la ordenanza, un grupo de productores agropecuarios iniciaron dos causas contra la Municipalidad para que la ordenanza fuera declarada inconstitucional ("Verdol S.A contra Municipalidad de Alta Gracia" y "Morardo, Julio Ángel y Otro contra Municipalidad de Alta Gracia"). Ante ello, los/as vecinos/as decidieron acudir a los/as abogados/as nucleados en la Clínica Jurídica de FUNDEPS (Fundación para el Desarrollo de Políticas Sustentables). De la mano de estos/as profesionales, primero presentaron un Amicus Curiae en 2013, con argumentos y evidencia que fortalecieran la postura de la Municipalidad a favor de la Ordenanza. Luego solicitaron ser aceptados/as como Terceros Interesados en la causa, lo cual fue admitido en 2015. Como partes intervinientes del caso, FUNDEPS y los/as vecinos/as pudieron acceder a la presentación de pruebas, así como a información actualizada sobre las etapas del juicio. 
Saccucchi, E. y Avila Castro, M.P. Análisis de discurso de la judicialización de cuatro conflictos ambientales en Córdoba, Argentina. Derecho y Ciencias Sociales. Mayo - Octubre 2020. № 23 .Pgs 1-22 ISNN 1852-2971. Instituto de Cultura Jurídica y Maestría en Sociología Jurídica. FCJ y S. UNLP

\subsubsection{Caso instalación de la empresa Monsanto en la localidad de Malvinas Argentinas}

En 2012 la empresa Monsanto se propuso construir en Malvinas Argentinas, a unos 12 kilómetros al este de la ciudad de Córdoba, una planta de separación, tratamiento, acondicionamiento y embolsado de semillas de maíz transgénico. La misma comenzó a construirse en un predio de 27 hectáreas ubicado sobre la ruta provincial A-188.

Un grupo de vecinos/as comenzó a reunirse y a problematizar la radicación de la planta, motivo por el cual decidieron conformar la Asamblea de Malvinas Lucha por la Vida. Tras casi un año de diversas acciones, en septiembre de 2013, militantes de la Asamblea, de Madres de Barrio Ituzaingó, vecinos/as autoconvocados/as y entidades sociales iniciaron el bloqueo del paso hacia el predio donde se construiría la planta. Asimismo, este conjunto de actores con el acompañamiento del Club del Derecho, presentó un amparo ambiental en septiembre de 2012 para la paralización de las obras de la empresa Monsanto hasta tanto no se cumplimentasen los diversos procedimientos aplicables en materia ambiental que establece la Ley General Nacional de Ambiente $\mathrm{N}^{\circ} 25.675$.

El amparo, primero fue rechazado por el Juzgado de Conciliación de Sexta Nominación, a cargo de Carlos Moroni, frente a lo cual los/as vecinos/as apelaron y en octubre de 2012, la Sala 2 de la Cámara del Trabajo les hizo lugar. Con ello se ordenó que se detuviera la obra. En enero de 2014 la Sala Segunda de la Cámara del Trabajo ordenó la suspensión de las obras hasta tener el Estudio de Impacto Ambiental y celebrar una audiencia pública. Un mes más tarde, la Secretaría de Ambiente junto al Ministerio de Agua, Ambiente y Energía, rechazaron el EIA presentado por Monsanto.

A su vez, en julio de 2016 la Justicia imputó a los funcionarios que habían habilitado la obra por abuso de autoridad. La imputación se dio a raíz de la investigación del fiscal Anticorrupción, Hugo Amayusco, hacia el ex intendente Arzani y el ex secretario de Ambiente de Córdoba, Luis Bocco. Entre los amparos, las demandas e informes realizados, así como por la tenacidad y perseverancia del bloqueo de Malvinas Lucha por la Vida en la puerta del predio de Monsanto, la empresa abandonó las tareas de la planta acondicionadora de semillas de maíz y vendió el predio a un privado.

\section{Análisis de las prácticas judiciales}

\section{1. El principio precautorio}

Uno de los argumentos discursivos más notorios en los procesos de lucha de las prácticas judiciales tiene que ver con el principio precautorio, el cual implica la obligatoriedad de 
Saccucchi, E. y Avila Castro, M.P. Análisis de discurso de la judicialización de cuatro conflictos ambientales en Córdoba, Argentina. Derecho y Ciencias Sociales. Mayo - Octubre 2020. № 23 .Pgs 1-22 ISNN 1852-2971. Instituto de Cultura Jurídica y Maestría en Sociología Jurídica. FCJ y S. UNLP

suspensión de toda actividad que presuntamente pueda tener un impacto grave en el medio ambiente. Las argumentaciones sostienen la idea de los derechos ambientales como derechos operativos, es decir, que deben ser inmediatamente aplicados y no requieren de una actividad legislativa que los reglamente.

La necesidad de operatividad en la tutela de los derechos ambientales debe ser entendida como un atributo de inmediatez que materializa el criterio precautorio; sólo disponiendo inmediata y expeditivamente el cese de los efectos dañosos de la actividad económica generadora del daño ambiental colectivo se evita su agravamiento. Esto supone la idoneidad del derecho ambiental como el único que, dadas estas características y herramientas nombradas, hace posible la pronta y eficaz resolución de la situación afectante. Cualquier otro remedio legal que se intente llegará tarde y será por tanto inútil a los fines buscados.

En el caso Monsanto, el Club de Derecho $^{2}$ señala entre los agravios de la instalación de la empresa en Malvinas Argentinas la transgresión a ciertos principios jurídicos ambientales, de acuerdo con lo prescripto por el artículo 4 de la Ley General de Ambiente $\mathrm{N}^{\circ}$ 25.675. Se trata de lo presentado en el amparo de 2013 en la causa "Club de Derecho (Fundación Club de Derecho Argentina) y otros -Quispe, Eduardo -Quispe Diego Raúl -Quispe Esther Margarita Molina Celina Laura -Barboza Vaca, Vanina de los Ángeles -Oliva, DA c/Municipalidad de Malvinas Argentinas -Amparo (Ley 4915) Expte: 218019/37”, el cual se presenta en tanto la planta supone diversos riesgos para la salud y el ambiente. El amparo indica que las semillas que serán almacenadas soportan glifosato y el glufosinato, productos que generarán altos niveles de contaminación y daño ambiental. A su vez, se señala que las actividades de la empresa tendrán consecuencias nocivas por el empleo en el curado de las semillas de gran cantidad de agua y la generación de una importante cantidad de líquidos residuales altamente peligrosos que la semillera debe desechar.

En particular, el amparo menciona el principio de prevención que establece que las causas y las fuentes de los problemas ambientales se atenderán en forma prioritaria e integrada, tratando de prevenir los efectos negativos que sobre el ambiente se pueden producir; y el principio precautorio, que dispone que, cuando haya peligro de daño grave e irreversible, la ausencia de información o certeza científica no deberá utilizarse como razón para postergar la adopción de medidas eficaces tendientes a impedir la degradación del ambiente en función de los costos.

\footnotetext{
${ }^{2}$ Organización sin fines de lucro y apartidaria, radicada en Córdoba (Argentina) que se dedica a la capacitación, actualización, formación y difusión del derecho y las ciencias sociales, en la comunidad jurídica y en la sociedad (http://www.clubdederecho.org).
} 
Saccucchi, E. y Avila Castro, M.P. Análisis de discurso de la judicialización de cuatro conflictos ambientales en Córdoba, Argentina. Derecho y Ciencias Sociales. Mayo - Octubre 2020. № 23 .Pgs 1-22 ISNN 1852-2971. Instituto de Cultura Jurídica y Maestría en Sociología Jurídica. FCJ y S. UNLP

Paradójicamente, en los casos bajo estudio las estrategias jurídicas analizadas que se sostienen en el principio precautorio van acompañadas de evidencia científica, a pesar de que el mismo no requiera de prueba alguna sino posibilidad de daño al ambiente y a la salud. En el caso de la Ordenanza de Alta Gracia, además del estudio sobre la población directamente afectada, el escrito de FUNDEPS ${ }^{3}$ incluye numerosos estudios que vinculan científicamente un número de enfermedades y daños ambientales con la aplicación sistemática de agrotóxicos en el esquema productivo de monocultivo de soja, a la vez que remarcan que "la incertidumbre científica no puede ser alegada como principios para la inacción del ente ejecutivo municipal, que efectivamente reguló porque entiende que existe un riesgo que 'con buenas razones, que puede llegar a existir"” (FUNDEPS y vecinos, Solicitud como Terceros Interesados).

En la solicitud presentada y como partes del caso, FUNDEPS citó el estudio que llevó adelante un conjunto de médicos/as especialistas del Hospital de Clínicas titulado "Informe del Estudio Epidemiológico Observacional realizado en B Parque San Juan de la Ciudad de Alta Gracia”, firmado por la Dra. Nelly Barrera. En este informe se destaca que del total de personas encuestadas el $51 \%$ estaba enfermo, siendo el asma la patología más frecuente en un 19,85\%, comprobado en 50 casos de los cuales 39 eran niños/as. También se hallaron con frecuencia otras afecciones como enfermedades endocrinas, cáncer, neurológicas, malformaciones congénitas (polidactilia, SD de Down, Ductus), enfermedades autoinmunes como lupus, miastenia gravis, celiaquía y afecciones obstétricas como abortos espontáneos, infertilidad.

En este mismo sentido, encontramos el caso de VUDAS contra Porta Hnos. En los amparos se citan diversos estudios científicos que señalan las evidencias de contaminación. En especial destacan tres estudios. Primero, la Auditoría Ambiental realizada por "SYMA Consultores" que realiza una descripción de las actividades productivas allí desarrolladas y las características técnicas de la planta. El mismo sostiene que la población más afectada en forma negativa es aquella que se encuentra en el entorno inmediato del predio.

Segundo, el Informe elaborado por la Cátedra Universitaria de Ambiente y Salud - Red de Médicos de Pueblos Fumigados denominado: “Análisis de la salud colectiva ambiental de barrio parque San Antonio. Impacto en la salud colectiva por contaminación de una planta de bioetanol". En este estudio se concluye que: "Nuestro diagnóstico confirma en la población del barrio San Antonio daño agudo y sostenido generado por contaminación química ambiental”.

\footnotetext{
${ }^{3}$ Organización sin fines de lucro, radicada en Córdoba (Argentina) que trabaja en la incidencia en políticas públicas a nivel local, nacional e internacional para que se respeten los derechos humanos. Realiza actividades de investigación, capacitación, incidencia, litigio estratégico y cooperación en general, para contribuir al desarrollo sustentable desde la protección de los grupos más vulnerables (http://www.fundeps.org).
} 
Saccucchi, E. y Avila Castro, M.P. Análisis de discurso de la judicialización de cuatro conflictos ambientales en Córdoba, Argentina. Derecho y Ciencias Sociales. Mayo - Octubre 2020. № 23 .Pgs 1-22 ISNN 1852-2971. Instituto de Cultura Jurídica y Maestría en Sociología Jurídica. FCJ y S. UNLP

A su vez, esta conclusión se apoya en la coincidencia de la identidad entre las patologías descritas y los efectos de exposición que surgen de las fichas Internacionales de Seguridad Química del Etanol.

En tercer lugar, se cita el trabajo denominado "Evaluación de material particulado y compuestos orgánicos volátiles en las inmediaciones de una fábrica de bioetanol" realizado por la Cátedra de Problemática Ambiental - Facultad Ciencias Exactas, Físicas y Naturales - (Universidad Nacional de Córdoba). Allí el equipo de investigación concluye: "En base a lo expuesto anteriormente podemos concluir, que los niveles de PM10 exceden las normativas vigentes de la OMS (...) Los compuestos orgánicos volátiles que se detectaron en el PM10 son tóxicos y peligrosos para la salud y el medio ambiente".

Por su parte, las y los vecinos de Malvinas Lucha por la Vida solicitaron a profesionales de la Universidad Nacional de Córdoba que analizaran la situación sanitaria de la población en la localidad. En 2012 se llevó adelante el “Análisis de la Salud Colectiva Ambiental de Malvinas Argentina- Córdoba. Una investigación socio- ambiental y sanitaria a través de técnicas cualitativas y relevamiento epidemiológico cuantitativo", un informe firmado por Ruderman, Cabrera Fasolis, Dozzo, Nota y Ávila Vázquez. El estudio reconoce que la ciudad de Malvinas Argentinas presenta el perfil de enfermedades que se reitera en las poblaciones expuestas a pesticidas aerolizados de la Argentina. En el informe se detalla el alto impacto encontrado en salud reproductiva, respiratoria y dermatológica y la distribución espacial de las mismas, incluyendo tumores y cánceres, con un gradiente mayor en las zonas más expuestas a los pesticidas. A su vez, indican que la vulnerabilidad social y económica de la población es de las más altas en la Provincia de Córdoba y la capacidad del Estado municipal de responder a la demanda de enfermedad es mínima. Por ello, el equipo de profesionales concluye que la población no está en condiciones de soportar un nuevo golpe en su salud ambiental, como consecuencia de la instalación de la planta de semillas de Monsanto.

Asimismo, junto con la Fundación para la Defensa del Ambiente (FUNAM) las y los vecinos presentaron un informe en el año 2014, el cual estudió la presencia de agrotóxicos en los pobladores de la localidad y arrojó que había agrotóxicos en el organismo del 70 por ciento. El estudio se basó en 10 casos testigo, y en siete de ellos se encontraron rastros de productos tóxicos. Entre ellos, residuos de plaguicidas como Aldrin, Dieldrin, DDT y Beta HCH (Cba24n).

Por último, en el caso de Madres de Barrio Ituzaingó Anexo también se registra la estrategia de evidenciar los impactos y daños en la salud a través de diversos estudios. Principalmente 
Saccucchi, E. y Avila Castro, M.P. Análisis de discurso de la judicialización de cuatro conflictos ambientales en Córdoba, Argentina. Derecho y Ciencias Sociales. Mayo - Octubre 2020. № 23 .Pgs 1-22 ISNN 1852-2971. Instituto de Cultura Jurídica y Maestría en Sociología Jurídica. FCJ y S. UNLP

destacamos el Informe elaborado por el Epidemiólogo Ariel Depetris, ex-coordinador designado por la Organización Panamericana de la Salud (OPS) el cual sostiene que, a partir de un análisis de suelo y agua, se registran diversas sustancias contaminantes que tienen impacto en la salud de las personas.

En síntesis, a pesar de que el principio precautorio de las leyes ambientales no exige que se compruebe la presencia de contaminación o daños al ambiente y su sola presunción de posibilidad debería habilitar al fallo favorable de los amparos ambientales, las organizaciones socioambientales han solicitado, promovido, difundido y/o realizado estudios científicos que evidencian los daños señalados, a lo largo de sus procesos y/o a través de sus representantes legales. Más allá de la contundencia del principio precautorio, el análisis de los casos señala que los colectivos en lucha se ven obligados a argumentar y probar la presencia de contaminación y su consecuente impacto en la salud a partir del recuento de la cantidad de enfermos y afecciones. Ello supone que se le asigne al discurso científico el poder de probar lo denunciado. Así, se han desarrollado diversos estudios e investigaciones, tanto por parte de los colectivos, como por los representantes de las actividades en discusión. En otras palabras, el conflicto se traslada también al ámbito científico dado el gran poder que este recurso supone en tanto discurso de verdad (Saccucci, 2019).

Encontramos que la práctica judicial que los colectivos llevan adelante por medio de sus representantes legales, en contra de la contaminación ambiental del agronegocio, está configurada por la necesidad estratégica de legitimar el discurso a favor del ambiente sano, por medio de la evidencia del saber científico. El mecanismo del principio precautorio es una práctica judicial que, al contrario de sustraerse, conlleva a la indagación científica de las causas contaminantes.

\subsection{Argumentos procedimentales}

En el marco de los casos y documentos que estudiamos, el universo discursivo de estrategias judiciales no sólo no se agota en el principio precautorio, sino que se ubica en otro orden argumentativo, el de las formas e instrumentos propios del derecho y la política ambiental, más que en el contenido de un aspecto ambiental o sanitario degradado. Entre estos se destacan en el análisis: la ausencia de estudios de impacto ambiental, la carencia de audiencias públicas y el incumplimiento de disposiciones de uso del suelo y habilitaciones.

\subsubsection{Ausencia de estudios de impacto ambiental}


Saccucchi, E. y Avila Castro, M.P. Análisis de discurso de la judicialización de cuatro conflictos ambientales en Córdoba, Argentina. Derecho y Ciencias Sociales. Mayo - Octubre 2020. № 23 .Pgs 1-22 ISNN 1852-2971. Instituto de Cultura Jurídica y Maestría en Sociología Jurídica. FCJ y S. UNLP

Uno de los principales argumentos sostenido por los colectivos en lucha es la ausencia de estudios de impacto ambiental. Los estudios de impacto ambiental (EIA) son el mecanismo legal por excelencia para prevenir los daños ambientales potenciales. En diversos antecedentes que sientan jurisprudencia, los EIA han sido concebidos como un procedimiento previo a la toma de decisiones, que sirve para registrar y valorar de manera sistemática y global todos los efectos potenciales de un proyecto, con el objeto de evitar desventajas para el medio ambiente. De este modo, la no realización previa de los EIA supone el incumplimiento de un requisito exigible por la ley que posee un sentido de protección de los derechos. Se trata de un incumplimiento en el procedimiento formal que invalida la radicación de las actividades en discusión y las vuelve ilegales. La realización de los EIA está claramente incluida en la Ley General de Ambiente $\mathrm{N}^{\circ} 25.675$ (arts. 11, 12 y 13) y en la Ley Provincial de Ambiente $\mathrm{N}^{\circ}$ 10.208 (del artículo 13 al 34, se legisla extensamente sobre este instrumento de gestión ambiental).

De acuerdo con el amparo ambiental presentado en el caso VUDAS contra Porta Hnos:

"Por la presente no se discute el carácter <contaminante> de las actividades desarrolladas en la Planta de Bioetanol PORTA HNOS, muy por el contrario, lo que aquí se discute es si, la Empresa tiene las habilitaciones nacionales para funcionar y si cumplió con el procedimiento previo de EIA. Que sin perjuicio de ello, y de manera subsidiaria, los amparistas desean poner de manifiesto el carácter contaminante de la actividad, al solo efecto de la adopción de -manera inmediata y urgente- de la medida peticionada (...)" (p. 57).

En este extracto se hace evidente que hay dos estrategias argumentativas: una propia del derecho ambiental (la denuncia de contaminación) y otra que responde a argumentos procedimentales. Entre estas dos estrategias se establece una relación de jerarquía ya que el argumento procedimental es el eje principal del amparo ambiental, mientras que la denuncia de contaminación aparece como subsidiaria. En otras palabras, se da cuenta de la ilegalidad del emprendimiento y se señala la contaminación a los fines de anclar el reclamo bajo la garantía del principio precautorio que supone una inmediatez en el accionar del Estado.

Así, se argumenta la perpetración de un delito ya que la ausencia de EIA supone desconocer y violar la ley nacional $\mathrm{N}^{\circ} 26.093$ de biocombustibles y la ley $\mathrm{N}^{\circ} 25.675$, ley general de ambiente (LGA en adelante).

En una línea similar, encontramos la denuncia de la falta de habilitaciones y permisos legales o bien la presencia de habilitaciones precarias para el funcionamiento de las distintas actividades. 
Saccucchi, E. y Avila Castro, M.P. Análisis de discurso de la judicialización de cuatro conflictos ambientales en Córdoba, Argentina. Derecho y Ciencias Sociales. Mayo - Octubre 2020. № 23 .Pgs 1-22 ISNN 1852-2971. Instituto de Cultura Jurídica y Maestría en Sociología Jurídica. FCJ y S. UNLP

Esto refuerza la idea de que estas actividades no cumplen con los requisitos legales, por lo cual se encuentran funcionando de modo ilegal. Un claro ejemplo de ello es la denuncia que realizan las vecinas de VUDAS sobre la falta de inscripción de la empresa en el registro nacional de productores de biocombustibles, haciendo posible que la empresa niegue y desmienta que produce bioetanol. Así, la denuncia señala la ilegalidad manifiesta de la empresa y su funcionamiento de modo clandestino.

En el caso de la instalación de la planta de Monsanto en Malvinas Argentinas, el debate sobre la existencia y la calidad del EIA también tiene un peso significativo, de la mano de otros aspectos administrativos en consideración. El Club de Derecho presentó un recurso de amparo en septiembre de 2012 en contra de la Municipalidad de Malvinas Argentinas, a partir del cual se solicitaba la paralización de la instalación de Monsanto y la declaración de inconstitucionalidad de la Ordenanza que había autorizado el inicio de obra. A su vez, se requería que no se realizara ningún tipo de autorización hasta tanto la empresa no presentara un EIA y la Municipalidad realizara una Consulta Popular. Lo que el equipo amparista señaló es que no se estaban cumplimentando los procedimientos que la normativa nacional sobre ambiente estipulaba para este tipo de emprendimientos, específicamente respecto de las obligaciones que emanan de la Ley General de Ambiente $\mathrm{N}^{\circ} 25.675$.

La discusión jurídica respecto del EIA de la empresa Monsanto no terminó en el amparo presentado en 2012. En 2014, y luego de sancionada la Ley de Política Ambiental de Córdoba $\mathrm{N}^{\circ} 10.208$, las condiciones administrativas respecto de este instrumento se modificaron. Por medio de un informe de la Asociación Argentina de Abogados Ambientalistas (AAdeAA), se cuestionó que la multinacional estuviera inclusive en condiciones de presentar un EIA, dado que la nueva ley expresa claramente que estos estudios no pueden presentarse por segunda vez luego de un rechazo (artículo 20). El dictamen de la AAdeAA fue entregado por la Asamblea Malvinas Lucha por la Vida al Ministerio de Agua, Ambiente y Servicios Públicos de Córdoba, para que el organismo tomara en consideración la imposibilidad de que la empresa volviera a entregar un EIA.

En cualquiera de los dos casos, queda en evidencia cómo el aspecto relativo a las formas y a los contenidos del estudio de impacto ambiental opera imposibilitando una aprobación de la radicación de la empresa Monsanto. La estrategia jurídica de las personas afectadas y sus representantes, el Club de Derecho o la AAdeAA, se estructura a partir de un argumento procedimental y su flagrante incumplimiento. En el caso Monsanto, además de las consideraciones respecto de las consecuencias nocivas para el ambiente y la salud por la 
Saccucchi, E. y Avila Castro, M.P. Análisis de discurso de la judicialización de cuatro conflictos ambientales en Córdoba, Argentina. Derecho y Ciencias Sociales. Mayo - Octubre 2020. № 23 .Pgs 1-22 ISNN 1852-2971. Instituto de Cultura Jurídica y Maestría en Sociología Jurídica. FCJ y S. UNLP

presencia de una planta como la que se proponía construir, es fundamental la invocación de instrumentos de gestión de política ambiental como el de los EIA.

\subsubsection{Ausencia de audiencias públicas}

Otro de los argumentos esgrimidos es la ausencia de audiencias públicas previas a la instalación de las actividades económicas en discusión. Si bien de acuerdo con la LGA las audiencias públicas no son vinculantes, representan un mecanismo de participación política de las comunidades involucradas. De acuerdo con el amparo ambiental de VUDAS contra Porta Hnos, la autoridad de aplicación no llevó adelante la audiencia pública que se requería previo a la decisión de instalación y desarrollo de actividades de la empresa.

De este modo, se abona la idea de la comisión de un delito en tanto no se ha cumplimentado con el requisito obligatorio de la audiencia pública y se ha lesionado el derecho de los/as ciudadanos/as a participar en la toma de decisiones. De acuerdo con este argumento que se enmarca en la LGA y en diversas normativas internacionales, la ciudadanía es clave en la "gestión" del medio ambiente. Es decir, deben ser sujetos activos en la conformación y administración de los proyectos de desarrollo locales y en su control, seguimiento y fiscalización. Para que dicha gestión pueda materializarse se deben poner en práctica los mecanismos de participación y consulta establecidos en la LGA. La ausencia de audiencias públicas privó a las/os ciudadanos de poder analizar de manera crítica, técnica y científica, los posibles impactos sobre el ambiente y la calidad de vida, impidiendo además mitigar o prevenir la ocurrencia del daño ambiental.

\subsubsection{Violaciones de las disposiciones del uso del suelo}

En los conflictos estudiados resulta frecuente encontrar argumentos que se basan en las disposiciones de los usos del suelo del Instituto de Planificación Metropolitana- IPLAM ${ }^{4}$ que define y regula los usos posibles de las diversas zonas y áreas metropolitanas vinculadas a Córdoba Capital. Precisamente los conflictos con el agronegocio que hemos seleccionado para este trabajo se localizan en territorios del Gran Córdoba (Alta Gracia, Malvinas Argentinas y zona sur de la ciudad de Córdoba), incluidos en las disposiciones del Plan de usos del suelo y

\footnotetext{
${ }^{4}$ El IPLAM fue creado en 2010 por medio de la Ley provincial 9.841, en el marco de un "Plan Metropolitano de Usos del Suelo", para un primer anillo alrededor de la capital. Se trata de una política pública provincial creada con el objetivo de planificar el crecimiento de la ciudad Capital y del denominado Gran Córdoba, a partir del establecimiento de las diversas posibilidades de uso del suelo en cada zona y se terminó de desarrollar con la ley 10.004 de diciembre de 2011 que ordena la segunda etapa.
} 
Saccucchi, E. y Avila Castro, M.P. Análisis de discurso de la judicialización de cuatro conflictos ambientales en Córdoba, Argentina. Derecho y Ciencias Sociales. Mayo - Octubre 2020. № 23 .Pgs 1-22 ISNN 1852-2971. Instituto de Cultura Jurídica y Maestría en Sociología Jurídica. FCJ y S. UNLP

en las políticas del IPLAM, las cuales se solapan con disposiciones propias del derecho ambiental.

Este argumento se destaca principalmente en el caso de la lucha en contra de la empresa de Monsanto y VUDAS contra Porta Hnos. En cuanto a este último, es preciso señalar que es un argumento esgrimido principalmente por las vecinas en sus discursos y que aparece en segundo plano en el amparo ambiental.

En el caso VUDAS contra Porta Hnos. las vecinas señalan que el barrio se encuentra emplazado desde la década de 1950 y que, por el contrario, la fábrica se radicó allí a partir de la década de 1990 a raíz de una “excepción” en el tipo de uso del suelo que pasaba a ser considerado mixto. Esta excepción fue tramitada por el padre del actual dueño de la empresa cuando aquel era Ministro de Industria. En el 2014, se sancionó la ordenanza 8133 que promulgaba la necesidad de relocalización de más de 200 industrias que se encontraban incumpliendo los usos de suelo dispuestos. Sin embargo, la empresa Porta Hnos. recibió una nueva extensión hasta la actualidad.

De allí que las vecinas reclamen por el incumplimiento de la norma de regulación de los usos posibles del suelo. A su vez, en el amparo ambiental presentado por ellas citan antecedentes de otras plantas productoras de bioetanol y sus respectivas localizaciones: la Planta de "ACABIO" (Villa María, Córdoba) ubicada a 3,5 km de la población urbana; la Planta "BIO 4" (Río Cuarto, Córdoba) a 2,46 km y la Planta "VILUCO S.A." (Frías, Santiago del Estero) a una distancia de 2,49 km de la población urbana.

La violación de las disposiciones de uso del suelo asume gravedad ante la posibilidad de explosión de la fábrica, no solo por la producción de bioetanol sino, incluso, por la producción de alcohol que es la actividad originaria de esta planta. Las vecinas han realizado un cálculo sobre la onda expansiva que podría tener la explosión: arrasaría la mitad de la ciudad.

El segundo argumento procedimental que se puso en juego en el caso de la instalación de la planta de acondicionamiento de semillas de maíz de la multinacional Monsanto, en la localidad de Malvinas Argentinas, se refirió al incumplimiento de las disposiciones del uso del suelo, de acuerdo con el mencionado Plan Metropolitano del IPLAM. La empresa se estaba radicando en un territorio considerado como área de actividad agropecuaria no contaminante, bajo la prohibición de actividades industriales como la de la planta. Así, la causa relacionada con “incumplimiento de deberes de funcionario público" y "abuso de autoridad” recayó en la Fiscalía Anticorrupción de Hugo Amayusco, quien comenzó a investigar, entre otros, al ex 
Saccucchi, E. y Avila Castro, M.P. Análisis de discurso de la judicialización de cuatro conflictos ambientales en Córdoba, Argentina. Derecho y Ciencias Sociales. Mayo - Octubre 2020. № 23 .Pgs 1-22 ISNN 1852-2971. Instituto de Cultura Jurídica y Maestría en Sociología Jurídica. FCJ y S. UNLP

Secretario de Ambiente del Gobierno de la Provincia Luis Federico Bocco y a autoridades del Municipio de Malvinas Argentinas.

En este sentido, no se invoca una figura de daño ambiental, sino el incumplimiento de las disposiciones de una ley de otro tipo, la Ley $\mathrm{N}^{\circ} 9.841$ que regula los usos del suelo del Gran Córdoba.

\section{Reflexiones finales}

En este artículo hemos presentado una aproximación teórica que permite comprender la creciente judicialización de los conflictos socioambientales. En las sociedades neoliberales, la dinámica de las empresas cobra protagonismo y debe desarrollarse sin intervención sobre las reglas de juego económicas por parte del poder político. En este contexto, el poder judicial asume centralidad como árbitro de los conflictos que pueden desencadenarse fruto del "libre" desarrollo de las empresas (Foucault, 2017b).

En particular, hemos encontrado que las estrategias jurídicas de los cuatro casos analizados se sustentan sobre argumentos propiamente ambientales -como el caso paradigmático del principio precautorio-, a la vez que sobre argumentos procedimentales -ausencia de EIA, ausencia de audiencias públicas y violación de las regulaciones de los usos del suelo-. En cada caso se establecen diversas correlaciones entre ambos tipos de argumentos.

Nuestra lectura de esta particular configuración de la práctica judicial en el marco del derecho ambiental es que en la demostración de ilegalidad de las actividades del agronegocio, interviene significativamente la legitimidad del discurso científico. Como asegura Villegas Guzmán, "en el caso de los conflictos socio-ambientales, el lenguaje científico-ambiental es parte de la argumentación legal” (2018, p.80). Cabe destacar que se trata de un lenguaje común en la elaboración de estrategias políticas y jurídicas, tanto a favor como en contra de los proyectos en disputa (Koberwein, 2018).

Asimismo, observamos que las prácticas judiciales no se circunscriben al ámbito del derecho ambiental. Por el contrario, en algunos casos han sido cruciales argumentos del derecho administrativo, del derecho político o del derecho urbanístico. Nuestro análisis de discurso sugiere, por lo tanto, que la práctica judicial en su dimensión polémica y estratégica pone en juego una variedad de argumentos provenientes de diversos ámbitos del derecho (o incluso que se encuentran por fuera de él cuando se apela a la evidencia científica epidemiológica).

Es lo que de Sousa Santos comprende como la interlegalidad que constituye la vida sociojurídica en la práctica, en la cual operan simultáneamente diferentes espacios y escalas jurídicas. 
Saccucchi, E. y Avila Castro, M.P. Análisis de discurso de la judicialización de cuatro conflictos ambientales en Córdoba, Argentina. Derecho y Ciencias Sociales. Mayo - Octubre 2020. № 23 .Pgs 1-22 ISNN 1852-2971. Instituto de Cultura Jurídica y Maestría en Sociología Jurídica. FCJ y S. UNLP

Ello es tan intenso que "al nivel de la fenomenología de la vida socio-jurídica, no se puede hablar de derecho y de legalidad, sino más bien de interderecho e interlegalidad" (de Sousa Santos, 2000, p.236). Un aspecto que este autor remarca cuando reflexiona sobre la interlegalidad es, precisamente, que la intersección de ámbitos jurídicos condiciona las redes de acciones en los procesos sociales. Cuando, por ejemplo, los sujetos en conflicto echan mano del derecho oficial y se movilizan entre diversas escalas o argumentos, manifiestan el carácter estratégico e instrumental de sus acciones.

Lo que vemos entonces es que los colectivos en lucha invocan un uso estratégico del derecho, más allá del reclamo por determinados delitos ambientales en sentido estricto. Este uso estratégico del derecho se sustenta tanto sobre el efecto legitimante que inviste a la evidencia científica, como sobre ciertos argumentos formales y administrativos o de regulaciones de otro tipo de materia no ambiental. Para los colectivos en lucha, el incumplimiento de estos aspectos puede presentarse de manera más clara y contundente frente a las formas de enunciación y apropiación ideológicas del derecho ambiental por parte de las autoridades jurídicas.

A partir de lo aquí analizado, se presentan nuevos interrogantes sobre cómo se construyen los discursos de saber-poder científicos, en el marco de qué relaciones específicas de poder y de qué modo se articulan configurando dispositivos.

\section{Bibliografía}

Alonso, M. C.; Avila Castro, M. P.; de la Vega, C. y Saccucci, E. (2015). Mapeando conflictos ambientales en Córdoba: principales actores, posiciones y estrategias. I Encuentro de Investigadores y Estudiantes en Ciencias Sociales y Humanidades y VI Encuentro de Investigadores de la Facultad de Ciencia Política y Relaciones Internacionales. Universidad Católica de Córdoba. Córdoba, del 5 al 6 de octubre. Disponible en: http://www2.ucc.edu.ar/facultades/ciencia-politica/publicaciones/primer-encuentro-deinvestigadores-y-estudiantes/

Azuela, A. (2006). Visionarios y pragmáticos: una aproximación sociológica al derecho ambiental. México: UNAM/Fontamara.

Barrera, N. (2012). Informe del Estudio Epidemiológico Observacional realizado en B Parque San Juan de la Ciudad de Alta Gracia. Córdoba: Hospital de Clínicas.

De la Vega, C. (2014). Conflictos por el territorio: las políticas mineras y la estabilización de fuerzas entre actores sociales. Los casos de Córdoba y La Rioja (tesis inédita de maestría). Maestría en Administración Pública. Instituto de Investigación y Formación en Administración Pública (IIFAP), Universidad Nacional de Córdoba.

de Sousa Santos, Boaventura. (2000). Crítica de la razón indolente. Contra el desperdicio de la experiencia. Volumen I. Bilbao: Desclée de Brouwer. 
Saccucchi, E. y Avila Castro, M.P. Análisis de discurso de la judicialización de cuatro conflictos ambientales en Córdoba, Argentina. Derecho y Ciencias Sociales. Mayo - Octubre 2020. № 23 .Pgs 1-22 ISNN 1852-2971. Instituto de Cultura Jurídica y Maestría en Sociología Jurídica. FCJ y S. UNLP

do Carmo, L. R. y Alvarez, M. F. (2009). Expansión del cultivo de soja, salud y medio ambiente. Situación en Córdoba (Argentina) y Mato Grosso (Brasil). Série Investigaciones (6), 183200 .

Downs, A. (1972). Up and Down with Ecology - The 'Issue-Attention Cycle'. Public Interest, $28,38-50$.

Entelman, R. (1982). Aportes a la formación de una epistemología jurídica en base a algunos análisis del funcionamiento del discurso jurídico. El discurso jurídico. Perspectiva psicoanalítica y otros abordajes epistemológicos, 86, 85-109.

Foucault, M. (2017a). La verdad y las formas jurídicas. Barcelona: Gedisa.

Foucault, M. (2017b). Nacimiento de la biopolítica. Curso en el Collège de France (19781979). Buenos Aires: Fondo de Cultura Económica.

Gutiérrez, R. (2015). Teoría y praxis de los derechos ambientales en Argentina. Revista Temas y Debates, 19(30), 13-36.

Harrison, K. (1996). Passing the Buck: Federalism and Canadian Environmental Policy. Vancouver: University of British Columbia Press.

Koberwein, A. (2018). Ciencia, derecho, política y cultura en el conflicto por el bosque nativo en la provincia de Córdoba, Argentina. Revista del Museo de Antropología, 11 (1), 217 228.

Landry, M. (2012). Corrientes críticas del derecho: un compromiso con la transformación social. Revista Filosofía del derecho, 67-87.

Offe, C. (1992). Partidos políticos y nuevos movimientos sociales. Madrid: Editorial Sistema.

Orlandi, E. P. (1994). Discurso, imaginário social e conhecimento. Aberto, 14(61), 2176-6673.

Peruzzotti, E. y Smulovitz, C. (2002). Controlando la política: ciudadanos y medios en las nuevas democracias latinoamericanas. Buenos Aires: Temas.

Ruderman, L., Fasolis, B. C., Dozzo, G., Nota, C., y Ávila Vazquez, M. (2012). Análisis de la Salud Colectiva Ambiental de Malvinas Argentina-Córdoba. Reporte inédito.

Universidad Nacional de Córdoba.

Saccucci, E. (2019). Los conflictos en torno al ambiente y los discursos científicos. RevIISE: Revista de Ciencias Sociales y Humanas, 13(13), 109-123.

Saccucci, E. (2018). La producción de territorios de sacrificio: un análisis de la lucha de VUDAS contra la empresa Porta. GOT: Revista de Geografia e Ordenamento do Território, (15), 363-386.

Sachs, W. (1996) Diccionario del desarrollo. Una guía del conocimiento como poder. Perú: PRATEC. 
Villegas Guzmán, S. M. (2018). Entre arenas movedizas: luchas por el acceso a la tierra y el ambiente en la provincia de Córdoba (Argentina). Nuestra Praxis. Revista de Investigación Interdisciplinaria y Crítica Jurídica, 75-88.

\section{Corpus de documentos legales}

Amparo ambiental colectivo presentado por integrantes de la Asamblea de Vecinos Unidos por la Vida (VUDAS) contra el Ministerio de Energía y Minería de la Nación.

Amparo ambiental colectivo presentado por Ávila Vázquez Medardo José Fidel contra Sres. Jorge Alberto Gabrielli y Francisco Rafael Parra.

Club de Derecho (Fundación Club de Derecho Argentina) y otros -Quispe, Eduardo -Quispe Diego Raúl -Quispe Esther Margarita -Molina Celina Laura -Barboza Vaca, Vanina de los Ángeles -Oliva, DA c/Municipalidad de Malvinas Argentinas -Amparo (Ley 4915) Expte: 218019/37. Año 2013.

Solicitud para ser tenido como Amicus Curiae de César Edgardo Murúa en representación de la Fundación para el Desarrollo de Políticas Sustentables (FUNDEPS), ante la Cámara en lo Civil y Comercial $\mathrm{N}^{\circ} 8$ de la ciudad de Córdoba, en el marco de la causa $\mathrm{N}^{\circ} 2388618$ / 36, autos caratulados "Verdol S.A. c/Municipalidad de Alta Gracia - Recurso de apelación". Año 2013.

Solicitud como Terceros Interesados de César Edgardo Murúa en representación de la Fundación para el Desarrollo de Políticas Sustentables (FUNDEPS) y vecinos de Alta Gracia, en el marco de la causa $\mathrm{N}^{\circ} 2388618$ / 36, autos caratulados "Verdol S.A. c/Municipalidad de Alta Gracia - Recurso de apelación". Año 2015. 